\title{
Melioidosis among returning workers in Bangladesh: imported or endemic?
}

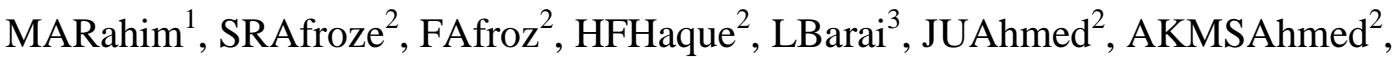 \\ MDHossain $^{2}$, MRRahman $^{2}$, AKMMusa $^{2}$, KNUddin $^{2}$
}

\section{Introduction}

In spite of significant sero-epidemiological surveillance reports and identification of Burkholderiapseudomallei from soil specimens, till date, only a few cases of melioidosis, mostly occurring in the north-east region of the country, have been reported in/from Bangladesh. We report four cases of melioidosis occurring among returning workers from endemic countries. Their home districts in Bangladesh were also reported as endemic for melioidosis. Our objective is to create awareness among physicians on the endemicity of melioidosis, both within and outside Bangladesh.

\section{Methods}

Patients' socio-demographic, clinical and laboratory data and treatment outcomes were recorded in case record forms.

\section{Results}

A 31-year-old plumber, newly detected as diabetic, returned to Bangladesh from Brunei with a diagnosis of septicaemic melioidosis with multiple hepatic and splenic abscesses and portal vein thrombosis. He initially presented with fever and chills. His initial treatment consisted of ceftazidime and amoxicillin-clavulanic acid combination was prescribed in continuation phase. Two months previously he had visited his home district Tangail, endemic for melioidosis in Bangladesh.

A 28-year-old diabetic man from Tangail, presented with fever and back pain. He was diagnosed as having spondylo-discitis and paravertebral abscess due to B. pseudomallei infection. Brucellosis and tuberculosis were excluded. He was treated with ceftazidime followed by cotrimoxazole and doxycycline. He used to work as carpenter in Saudi Arabia and returned home three months previously.

A 43-year-old newly detected diabetic carpenter, returning from Brunei, presented with threemonth history of recurrent fever, cough, sputum production and weight loss. He had been treated for pneumonia in Brunei and anti-tuberculosis medication was prescribed empirically at Brahmanbaria, his native district in Bangladesh, without much benefit. Subsequently, sputum

\footnotetext{
${ }^{I}$ Department of Nephrology, Bangladesh Institute of Research and Rehabilitation in Diabetes, Endocrine and Metabolic Disorders (BIRDEM) General Hospital, Dhaka, Bangladesh

${ }^{2}$ Department of Internal Medicine, Bangladesh Institute of Research and Rehabilitation in Diabetes, Endocrine and Metabolic Disorders (BIRDEM) General Hospital, Dhaka, Bangladesh

${ }^{3}$ Department of Microbiology, Bangladesh Institute of Research and Rehabilitation in Diabetes, Endocrine and Metabolic Disorders (BIRDEM) General Hospital, Dhaka, Bangladesh

Address for correspondence: Dr MA Rahim, Department of Nephrology, Bangladesh Institute of Research and Rehabilitation in Diabetes, Endocrine and Metabolic Disorders (BIRDEM) General Hospital, Dhaka, Bangladesh +88 1674534500 Email: muradrahim23@yahoo.com 1 https://orcid.org/0000-0003-0974-5730
} 
culture grew B. pseudomallei and he recovered with ceftazidime followed by doxycycline and co-trimoxazole.

A 37-year-old diabetic construction worker presented with non-resolving pneumonia for two months. He came back to Tangail three months ago from Singapore. Tuberculosis was excluded and blood culture grew B. pseudomallei. He was treated with meropenem followed by amoxicillin-clavulanic acid combination with complete resolution.

\section{Discussion and Conclusions}

Melioidosis is endemic in Bangladesh. Any patient with fever should raise suspicion of melioidosis, especially if diabetic and from endemic districts of Bangladesh or returning from endemic countries. 